CAHIERS DE

NARRATOLOGIE

\section{Cahiers de Narratologie}

Analyse et théorie narratives

37 | 2020

Approches transmédiales du récit dans les fictions contemporaines

\title{
Survie et transmission du monde perdu (Verne, Doyle, Cooper) : roman, vecteurs mémoriels, mythologies modernes
}

\section{Maxime Prévost}

\section{(2) OpenEdition}

\section{Journals}

Édition électronique

URL : http://journals.openedition.org/narratologie/10537

DOI : 10.4000/narratologie.10537

ISSN : 1765-307X

Éditeur

LIRCES

Référence électronique

Maxime Prévost, «Survie et transmission du monde perdu (Verne, Doyle, Cooper) : roman, vecteurs mémoriels, mythologies modernes », Cahiers de Narratologie [En ligne], 37 | 2020, mis en ligne le 04 septembre 2020, consulté le 06 septembre 2020. URL : http://journals.openedition.org/narratologie/ 10537 ; DOI : https://doi.org/10.4000/narratologie.10537

Ce document a été généré automatiquement le 6 septembre 2020

Article L.111-1 du Code de la propriété intellectuelle. 


\title{
Survie et transmission du monde perdu (Verne, Doyle, Cooper) : roman, vecteurs mémoriels, mythologies modernes
}

\author{
Maxime Prévost
}

1 Cette mise au point théorique prendra pour exemple la caution donnée par le collectif anonyme à une topique qui, en quelques décennies, s'est transformée en mythologie : celle du « monde perdu », c'est-à-dire cette topique à l'origine romanesque (elle naît en 1864 dans Voyage au centre de la terre) du monde primordial, enfoui, oublié, débouchant sur un temps différant radicalement du temps historique qui est le nôtre, topique qui, dans les décennies suivant la première réception de l'œuvre de Jules Verne, se constitue avec tout le caractère de l'évidence propre aux mythologies modernes, transitant notamment par The Lost World d'Arthur Conan Doyle (1912) et King Kong de Merian C. Cooper (nous considérerons ici comme un tout le film de 1933 et sa «novélisation » qui le prédate d'un an).

2 "Le mythe révèle", selon Pierre Brunel (1988: 8-9); il est "l'objectivation de l'expérience sociale de l'humanité ", écrivait Ernst Cassirer (1993: 72), et, selon l'herméneutique de la question et de la réponse chère à l'École de Constance (et dont certains aspects des écrits de Cornelius Castoriadis et de Hans Blumenberg se rapprochent, comme nous le verrons), il se montre particulièrement révélateur par sa survie, c'est-à-dire par sa capacité à se prêter à un questionnement renouvelé. Qu'estce que révèle et objective la mythologie des mondes perdus, et par quelles voies ce qui est à l'origine «le phantasme privé d'un individu exceptionnel» (pour citer Cornelius Castoriadis ${ }^{1}$ ) parvient à pénétrer l'imaginaire social, puis collectif ? 


\section{Mythes et mythologies modernes}

3 Commençons par des questions de définition ${ }^{2}$. Comme le souligne Michel Angot, le mot mythologie revêt au moins deux sens distincts : mythologie I : « le discours des Anciens dans leur propre langue destiné à leurs contemporains » et mythologie II : « le discours des Modernes dans leur langue sur la mythologie $I^{3} »$ (Angot 2019 : 16). À ces deux sens pourrait s'ajouter un troisième, appelons-le mythologie III : le discours des Modernes, dans leur langue, sur leurs inventions culturelles dont la constitution, avalisée par la collectivité, devient institution, c'est-à-dire représentation collective et non religieuse, procédant d'une forme de panthéon laïc.

Le mythe moderne, ou le mythe vivant, est un personnage (ou parfois un lieu - comme l'île au trésor - ou encore une donnée d'intrigue - le tour du monde en quatre-vingts jours; ces deux derniers pouvant se fondre, comme dans La Planète des singes) dont la célébrité et le rayonnement transcendent ceux de leur créateur - ou de leurs créateurs (car la mythologie est plurielle), lesquels peuvent rapidement en venir à se sentir dépossédés de leur création, voire menacés par elle ${ }^{4}$. C'est dire que l'imaginaire social d'une époque donnée s'ouvre sur un panthéon laïc de personnages issus de la fiction et des arts de représentation. Pour "authentifier " un mythe moderne, il faut ensuite tenir compte de sa longévité : le mythe s'inscrit dans la durée, et en vient à caractériser l'imaginaire de plusieurs générations. Il était possible de croire, en 1882, comme l'écrivait alors Maupassant, qu'il ne resterait rien de Dumas père, une fois son fils disparu; ce ne l'était plus en $1982^{5}$. Les mythologies sont quant à elles plus diffuses : si Superman est un mythe moderne, on peut parler de mythologies du superhéros pour évoquer l'univers fictionnel, familier à tout un chacun, dans lequel se déploient les justiciers surpuissants depuis l'apparition de ce personnage dans les pages d'Action Comics en juin $1938^{6}$. Si le personnage du capitaine Nemo, ou l'idée d'un tour du monde en quatre-vingts jours, sont des mythes, l'imaginaire des mondes perdus tient plutôt de la mythologie. Jules Verne est en effet un remarquable pourvoyeur de telles mythologies. Car, comme l'observe Brian Taves, les mythologies verniennes ne sont que rarement fondées sur les personnages en tant que tels ${ }^{7}$, c'est-à-dire qu'aucun personnage absolument central de notre panthéon laïque n'est spontanément associé à son nom, comme Tarzan à celui d'Edgar Rice Burroughs, Dracula à celui de Bram Stoker ou Sherlock Holmes à celui d'Arthur Conan Doyle. En effet, il s'impose davantage comme un pourvoyeur de mythologies que de mythes modernes (encore que les personnages du capitaine Nemo et de Phileas Fogg jouissent d'un rayonnement considérable, particulièrement dans la sphère anglophone pour ce dernier). Parmi ces mythologies vivantes, celle du "monde perdu » se distingue par sa vitalité; Voyage au centre de la terre "constitue ${ }^{8}$ " la topique du monde primordial, enfoui, oublié, débouchant sur un temps hors de l'histoire. Cette topique, qu'exploitera aussi le mythographe aujourd'hui méconnu qu'est Henry Rider Haggard (lequel s'intéressera plus précisément aux civilisations perdues - et retrouvées dans la fiction), réalise une grandiose synthèse entre le roman historique et ce roman géographique qui, selon Jean-Marie Seillan (2008 : 199-218), était destiné à le supplanter dans la seconde partie $\mathrm{du}$ siècle. Roman fondamental du " merveilleux géographique ", "Voyage au centre de la terre est à ce titre un chef-d'œuvre du voyage dans l'espace et le temps qui procède d'une véritable géographie cachée » (Dupuy 2013 : 59-60). 


\section{L'objectivation de l'expérience sociale de l'humanité}

5 Revenons à cette citation lumineuse extraite du Mythe de l'État d'Ernst Cassirer : "Le mythe constitue l'objectivation de l'expérience sociale de l'humanité. » Objectivation de quoi ? objectivation comment? Proposons ici un détour par l'herméneutique de la question et de la réponse de Hans Robert Jauss. «Le mythe révèle », écrivait donc Pierre Brunel, et, selon cette herméneutique de la question et de la réponse', il se montre particulièrement révélateur par sa survie, c'est-à-dire par sa capacité à se prêter à un questionnement renouvelé. Cette forme d'herméneutique, théorisée par Jauss dans Pour une herméneutique littéraire à partir des dialogues implicites que se livrent les auteurs à travers les âges autour de figures de la mythologie classique ou médiévale, doit être maniée avec précaution pour qui s'intéresse davantage à l'imaginaire et l'histoire culturelle qu'à celle des prétendus chefs-d'œuvre de la littérature, car, pour s'en tenir à un exemple clair, rien n'indique que le Mon Faust de Paul Valéry ait été avalisée par le collectif anonyme.

Observons qu'une telle heuristique n'est aucunement exclusive à Jauss; il est frappant de constater que d'autres penseurs, qui ont développé leurs théories de manière simultanée mais tout à fait indépendante des siennes, soient arrivées à des conclusions, voire à des méthodes, partiellement semblables. Songeons ici à Cornelius Castoriadis et à ce passage de L'Institution imaginaire de la société où il observe que :

Toute société jusqu'ici a essayé de donner une réponse à quelques questions fondamentales : qui sommes-nous, comme collectivité ? que sommes-nous, les uns pour les autres? où et dans quoi sommes-nous? que voulons-nous, que désironsnous, qu'est-ce qui nous manque? La société doit définir son "identité », son articulation; le monde, ses rapports à lui et aux objets qu'il contient; ses besoins et ses désirs. Sans la "réponse » à ces "questions », sans ces "définitions ", il n'y a pas de monde humain, pas de société et pas de culture - car tout resterait chaos indifférencié. Le rôle des significations imaginaires est de fournir une réponse à ces questions, réponse que, de toute évidence, ni la «réalité » ni la « rationalité » ne peuvent fournir [...]. Les questions ne sont même pas posées préalablement aux réponses. La société se constitue en faisant émerger une réponse de fait à ces questions dans sa vie, dans son activité. C'est dans le faire de chaque collectivité qu'apparaît comme sens incarné la réponse à ces questions, c'est ce faire social qui ne se laisse comprendre que comme réponse à des questions qu'il pose implicitement lui-même. (Castoriadis 1975 : 221)

Bien sûr, le maniement des théories de Castoriadis est tout aussi délicat, celui-ci ne parlant pas de littérature ni, de manière générale, d'art de représentation; je proposerai toutefois de considérer les mythes modernes (ou vivants) comme partie intégrante de ce faire par lequel la société apporte des réponses à ses interrogations fondamentales, et ce, dans la modernité, hors du cadre des religions institutionnalisées. Ces considérations mènent naturellement à penser que, parmi les trois fonctions que Pierre Brunel (1988 : 9) attribue au mythe (raconter, expliquer, révéler), la troisième est sans doute la plus captivante pour qui s'intéresse à l'aspect social des représentations, surtout celles qui parviennent à s'inscrire dans la durée.

Postulons donc que les mythes et mythologies modernes apportent des éléments de réponse à des questionnements sociaux, latents (dans la mesure où le mythe moderne apporte une réponse à une question dont on n'avait pas conscience qu'elle se posait) mais historiquement déterminés (et c'est dans ce sens qu'il y aurait objectivation de l'expérience sociale). On lit chez Hans Blumenberg: 
En ce qui concerne le potentiel d'efficience du mythe, il est essentiel de bien voir ceci : ce n'est pas la force de conviction de réponses anciennes à des énigmes prétendument intemporelles de l'humanité qui fonde la persistance insistante des configurations mythiques, mais plutôt l'existence implicite en elles de questions qui sont découvertes, dégagées et articulées dans leur réception et dans le travail qui s'accomplit sur elles. Que les mythes cosmogoniques possèdent encore un pouvoir de fascination à l'époque des cosmogonies théoriques ne tient pas à la réponse qu'ils formulent à la question théorique sur l'origine du monde, mais plutôt au surgissement de questions aussi puissantes qu'élémentaires qu'une théorie de l'origine du monde laisse sans réponse [...]. (Blumenberg 2005 : 69-70)

C'est en somme dire que l'œuvre qui parviendra à s'instituer en mythe ou se décliner en mythologies offre, au cours de son histoire, différentes réponses à des questions nouvelles, qui surgissent au fil de sa réception. On pourra ainsi analyser selon différentes perspectives les moments de de sa réception : son entrée dans un imaginaire social précis, c'est-à-dire celui du moment de sa première réception, une analyse sociocritique pouvant ainsi objectiver l'adéquation d'une œuvre donnée et de son contexte (et cotexte ${ }^{10}$ ) de production. La mythologie passant de génération en génération s'affranchit de cet imaginaire social synchronique pour entrer dans la diachronie de l'imaginaire collectif, apportant parfois de nouvelles réponses à de nouveaux questionnements sur le long terme ; la perspective mythocritique serait celle de ce dévoilement, cherchant à retracer et comprendre cet historicité passant de la constitution à l'institution d'une pierre de touche culturelle et imaginaire, demeurant constamment sensible aux écarts, à l'intrusion du nouveau dans ce qui est déjà là.

\section{Collectif anonyme et vecteurs mémoriels}

10 Naturellement, toute réflexion sur le mythe est une réflexion sur le collectif, sur ce que Castoriadis appelle le "collectif anonyme ${ }^{11}$ ", sur les voies de la constitution et de l'institution de l'imaginaire, attendu que l'œuvre qui s'instituera en imaginaire social, puis collectif est forcément une œuvre collective, conçue par plusieurs créateurs dont certains sont voués à demeurer anonymes, mais encore et surtout parce que c'est la collectivité qui pourra activer (ou non) son potentiel de "mythisme», sans quoi l'œuvre demeure le fantasme privé d'un créateur solitaire. Le mythe ou la mythologie s'institueront en outre par le biais de vecteurs mémoriels prenant le relais de l'œuvre de sa première exposition.

11 Qu'est-ce qu'un vecteur mémoriel ${ }^{12}$ ? Considérons comme vecteur mémoriel toute adaptation, tout relais, d'une œuvre donnée qui assure à la fois sa survie et son renouvellement. Les vecteurs mémoriels sont renouvelables et démodables: ils sont appelés à être remplacés au fil des décennies, le Sherlock Holmes de Benedict Cumberbatch se superposant à ceux de Basil Rathbone et de Jeremy Brett. Je dis bien se superposant car, comme l'écrivait Hans Blumenberg: "Dans le mythe, on n'est pas confronté à des décisions, il n'exige pas de renoncements» (2005: 28). Certains personnages, lieux imaginaires, données d'intrigue pénètrent donc à court terme l'imaginaire social, à long terme l'imaginaire collectif par l'entremise d'une adaptation marquante: Carmen est davantage la fille de Bizet, Meilhac et Halévy que celle de Prosper Mérimée; le Dracula que visualise spontanément le collectif anonyme depuis les années 1930 ressemble à celui qu'interprète Bela Lugosi dans l'adaptation de Tod 
Browning; la planète des singes est mieux connue par l'adaptation cinématographique de Franklin J. Schaffner que par le roman de Pierre Boulle.

La survie de l'œuvre de Jules Verne, comme celle de tout mythographe, est forcément collective : pour le dire comme Cornelius Castoriadis, son fantasme personnel ne se constitue en imaginaire social que tel qu'avalisé par le collectif anonyme. Jules Verne après Jules Verne est le résultat d'un immense et chaotique dialogue entre chacun des avatars culturellement significatifs de son œuvre et les romans d'origine, lesquels tendent aujourd'hui à redevenir leur propre vecteur mémoriel, en l'absence d'adaptation satisfaisant la collectivité et à cause du vieillissement de ses principales adaptations. Jules Verne après Jules Verne, c'est encore et surtout la migration des mythologies qu'il constitue ou qu'il infléchit vers d'autres supports, d'autres œuvres, d'autres auteurs laissant à leur tour une profonde empreinte culturelle. Dans le cas qui nous occupe, le Voyage au centre de la Terre survit et s'actualise à travers The Lost World et King Kong.

\section{Voyage au centre de la terre}

13 Nous en arrivons donc à la mythologie des mondes perdus. Que peut révéler et objectiver cette mythologie? Précisons d'abord ses contours. Elle prend vraisemblablement son origine, ou $\mathrm{du}$ moins trouve sa première exposition historiquement déterminante, dans le Voyage au centre de la terre de Jules Verne, en 1864. Dans ses Fragments d'un journal, Mircea Eliade résumait ainsi l'impression que lui avait laissée cette lecture :

Je lis le Voyage au centre de la terre de Jules Verne, et je suis fasciné par la hardiesse des symboles, la précision et la richesse des images. L'aventure est proprement initiatique et comme dans toute aventure de cet ordre, on trouve les égarements à travers le labyrinthe, la descente au monde souterrain, le passage des eaux, l'épreuve du feu, la rencontre avec les monstres, l'épreuve de la solitude absolue et des ténèbres, enfin, l'ascension triomphante qui n'est autre que l'apothéose de l'initié. Comme elles sont justes, les images de ces mondes souterrains - les autres mondes-, admirablement précise et cohérente aussi la mythologie à peine camouflée par le jargon scientifique de Jules Verne. Comment les psychologues et les critiques littéraires ont-ils pu ignorer jusqu'ici ce document exceptionnel, cet inépuisable trésor d'images et d'archétypes. Quelqu'un écrira bien, un jour, l'histoire de l'imagination moderne. Le chapitre consacré aux mondes souterrains devra tenir compte du Voyage au centre de la terre, de She par Rider Haggart [sic], de Om et de There was a door par Talbot Murphy. Aucun de ces trois auteurs n'entendait sans doute grand-chose aux mythologies, ni aux rites initiatiques. Et cependant les images, l'agencement des scènes qui évoquent les mondes souterrains et qui abondent dans leurs livres ne révèlent leurs significations secrètes que si on les réintègre dans le mystère dont elles ont été détachées, dans ce mystère inhérent à toute initiation? (Eliade $1973: 232$ )

14 Le «centre de la Terre» vernien semble correspondre à celui des époques préhistoriques: "Nous observons une très forte corrélation entre la distance parcourue à l'intérieur de la terre et le retour dans le temps » (Dupuy 2013: 7). Et, parmi les éléments énumérés par Eliade, il semble que la rencontre avec les monstres (préhistoriques) ait été particulièrement porteuse de fascination collective. En effet, Verne fait des entrailles terrestres le lieu où s'incarnent « les merveilleuses hypothèses de la paléontologie » (2016 [1864] : 180), le lieu où la préhistoire se fond dans l'histoire. D'abord dans le Chapitre xxxII, consacré au «rêve d'Axel », chapitre-vision dans lequel 
la pêche de poissons primitifs ( «une grande quantité de Pterychtis, ainsi que des poissons appartenant à une famille également éteinte, les Dipterides $\left.{ }^{13} »\right)$ provoque une méditation sur les états antérieurs de la planète ( $«$ Les siècles s'écoulent comme des jours! Je remonte la série des transformations terrestres $\left.{ }^{14} »\right)$. Ensuite et surtout, dans le Chapitre XxxIII, où l'on découvre que ce temps primordial subsiste effectivement dans les entrailles terrestres, Axel, le professeur Lidenbrock et leur guide Hans assistant au combat titanesque entre un "ichthyosaurus " et un "plesiosaurus» (Verne 2016 [1864] : 188 et suiv.). La mythologie des monde perdus postule la coexistence du monde moderne, largement désenchanté, et celle de mondes primordiaux, enfouis, oubliés ; autrement dit, elle fait coexister dans un même continuum les temps historique et mythique.

Notons que se constitue aussi une mythologie parallèle des civilisations perdues, celle-ci trouvant sans doute son exposition déterminante dans l'œuvre de Henry Rider Haggard ${ }^{15}$. Le protagoniste du roman She découvrira au cœur de l'Afrique la trace de civilisations prédatant celle de l'Égypte, qui serait le résultat tardif d'une migration vers le Nord africain. "[I]t seems that the world is very old", dit le professeur Holly à She, permettant ainsi à la déesse de compléter son éducation: "Old? Yes, it is old indeed. Time after time have nations, ay, and rich and strong nations, learned in the arts, been and passed away and been forgotten, so that no memory of them remains " (Haggard 2001 [1887] : 183). Tant dans le Voyage au centre de la Terre que dans She une vingtaine d'années plus tard, le roman s'impose comme lieu de coexistence du terre à terre et de l'inouï, coexistence qui sera centrale aux deux premiers vecteurs mémoriels de la mythologie des mondes perdus: The Lost World d'Arthur Conan Doyle (1912) et King Kong (1933).

\section{The Lost World et King Kong}

16 "You are a Columbus of science who has discovered a lost world", dit le journaliste Malone au professeur Challenger d'Arthur Conan Doyle (2001 [1912] : 41). Observons tout d'abord que la figure du professeur-aventurier est l'une des composantes de la mythologie constituée par Jules Verne, du professeur Lidenbrock au professeur Challenger en passant par le professeur Holly de Henry Rider Haggard. Aussi est-il significatif qu'à ce personnage, dans King Kong, se substitue la figure du cinéasteaventurier - nous y reviendrons. Tel le professeur Lidenbrock, Challenger, bien conscient du fait que les espaces inexplorés se raréfient («The big blank spaces in the map are all being filled in, and there's no room for romance anywhere »), découvre malgré tout un lieu de survie de la préhistoire, non pas au centre de la terre cette foisci, mais dans un plateau quasi inaccessible de l'Amérique du Sud. Le professeur et ses acolytes aventuriers y découvrent "un marais aux ptérodactyles", des iguanodons, ichtyosaures et autres tyrannosaures mangeurs de chair. Le professeur Challenger songe qu'il est heureux que l'humanité n'ait jamais eu à cohabiter avec ces créatures qui l'auraient sinon exterminée ${ }^{16}$. Même nos armes modernes, songe Challenger, ne font tout simplement pas le poids face aux mastodontes de la création primitive, constatation qui fait écho à cette notation du Axel du Voyage au centre de la Terre : «Le monde appartenait alors aux reptiles. Ces monstres régnaient en maitres sur les mers jurassiques. La nature leur avait accordé la plus complète organisation. Quelle gigantesque structure! quelle force prodigieuse! Les sauriens actuels, alligators ou 
crocodiles, les plus gros et les plus redoutables, ne sont que des réductions affaiblies de leurs pères des premiers âges! » (2001 [1912] : 185-186).

Le questionnement que semble objectiver cette mythologie en voie d'institution concernerait ainsi l'existence sécuritaire : l'être humain est-il encore à la hauteur du sublime de sa planète, dans un monde où le mystère et les espaces vierges sur les cartes géographiques se raréfient? On le voit, la mythologie du monde perdu met à mal ce qui passe de nos jours pour des impératifs catégoriques : la recherche de la sécurité et du confort. Jules Verne et Conan Doyle, comme tous les autres grands romancers, rappellent aux lecteurs du tournant du siècle que le confort et la sécurité ne constituent pas des valeurs, et que notre monde sécuritaire et confortable est un monde désenchanté. Tel est le sens, génialement humoristique, de la trajectoire initiatique du jeune journaliste Malone qui accompagne le professeur dans son périple : il est lancé à l'aventure par la jeune femme qu'il courtise, laquelle lui fait comprendre que seul un homme se modelant sur les grands explorateurs de l'Afrique des générations antérieures pourra la séduire : «It is never a man that I should love, but always the glories he had won. For they would be reflected upon me. Think of Richard Burton!» (2001 [1912] : 12). Cette Gladys Hungerton résume le tout en une formule prenante : "There are heroisms all around us waiting to be done " (2001 [1912]:12); gonflé à bloc, le jeune homme se lance donc à la recherche du monde perdu ; de retour à Londres, il la retrouve mariée à un greffier de notaire, filant le parfait bonheur dans une union petite-bourgeoise. Peu lui importe, car sa vie a désormais un sens; il sait que le monde atteint la hauteur du romanesque (« Apparently, the age of romance was not dead, and there was common ground upon which the wildest imaginings of the novelist could meet the actual scientific investigations of the searcher for truth ${ }^{17}$. »). Le monde serait vaste, dépaysant, dangereux, sublime au sens de Burke ${ }^{18}$, et la littérature aurait pour fonction de nous le rappeler à travers des fictions à caractère mythologique se détournant volontairement de la vraisemblance, privilégiant l'exploit à l'exploration psychologique ou sociologique, des fictions, donc, dont la force élémentaire s'apparenterait davantage à la psychologie des profondeurs ou la mise en récit d'archétypes qu'à quelque forme de concurrence à l'état civil.

18 King Kong est la parfaite illustration de la nature collective de la mythographie ; avant même d'être avalisé par le collectif anonyme, ce gigantesque gorille est d'emblée une création collective : on peinerait à identifier un seul nom à sa création, comme c'est aussi le cas pour les mythes de Robin des Bois ou de Zorro. La «novélisation » de King Kong, écrite à partir du scénario par Delos W. Lovelace, est publiée à la fin 1932, quelques semaines avant le film de 1933, et lui sert donc en quelque sorte de publicité. King Kong, comme une bonne part des mythes modernes, est le fils de plusieurs pères : le producteur Merian C. Cooper, d'abord, qui rêvait de raconter une histoire de gorille géant depuis qu'il avait lu Paul du Chaillu (Adventures in the Great Forest of Equatorial Africa and the Country of the Dwarfs, New York, Harper \& Brothers, 1890), et plusieurs scénaristes, dont le romancier Edgar Wallace, qui malgré le rôle mineur qu'il a joué dans la conception du scénario final (il en avait réalisé une ébauche préparatoire), a vu son nom attaché au film et au livre, à cause de sa célébrité dans les années 1930 ; les deux scénaristes qui apparaissent au générique sont finalement James Creelman et Ruth Rose. Ajoutons le nom du coproducteur et (avec Cooper) coréalisateur : Ernest B. Schoedsack. On pourrait ajouter l'animateur Willis O'Brien, qui s'était fait remarquer de Cooper par des animations pour une adaptation du Lost World de Conan Doyle ${ }^{19}$. 
Le personnage de Carl Denham est ici l'héritier de Lindenbrock et de Challenger ; non plus un professeur, donc, mais bien un cinéaste, ce qui semble objectiver le recul de la figure du scientifique, le monde se dévoilant désormais moins par son exploration ou sa description que par sa monstration. De même, le roman semble devoir céder le pas au film, le cinéma devenant désormais le principal véhicule du romanesque. Comprenant qu'il se trouve sur une île où survivent vraisemblablement les monstres préhistoriques, Denham se dit avec tout l'enthousiasme de l'évidence : « Why shouldn't such an out-ofthe-way spot be just the place to find a solitary, surviving prehistoric freak ? (Wallace, Cooper et Lovelace 2005 [1932] : 62). On pourrait considérer King Kong, ce monstre tout droit sorti de la préhistoire, comme la cristallisation de la mythologie des mondes perdus, celle qui va désormais se propulser de décennie en décennie. Nous nous arrêtons ici en 1933, mais l'étude resterait à faire des différentes itérations de ce que deviendra le mythe de King Kong, en particulier les adaptations cinématographiques de $1976^{20}, 2005^{21}$ et $2017^{22}$, tant de vecteurs mémoriels objectivant sa malléabilité et sa réceptivité à de nouveaux enjeux (par exemple à la militarisation de la société américaine, pour ce qui est de la dernière version en date, alors que le version de Peter Jackson proposait une réflexion cauchemardesque sur le devenir de la virilité en temps de crise économique ${ }^{23}$ ). En effet, avec King Kong, la mythologie du monde perdu s'incarne dans un mythe, c'est-à-dire dans un personnage mythique, tout comme la mythologie du vampire s'est faite mythe avec Dracula, ou comme la mythologie du détective s'est cristallisée dans le personnage de Sherlock Holmes. Il serait tentant d'en conclure que les mythologies, pour se propulser efficacement de génération en génération, doivent se faire mythe, c'est-à-dire s'incarner en un personnage surchargé de potentiel heuristique, qui deviendra l'agent de projections, de désirs, de craintes et de questionnements renouvelés au fil des générations. Tout comme Sherlock Holmes ou Dracula, King Kong est un tel personnage, «objectivation de l'expérience sociale de l'humanité » en cela qu'il objective, pourrait-on dire, les processus parallèles de civilisation des mœurs et de désenchantement du monde, retour, donc, d'un refoulé objectivement présent dans l'imaginaire collectif, mais difficilement perceptible sans le recours à la mythocritique.

\section{BIBLIOGRAPHIE}

Angot, Michel (2019), Les Mythes des Indes, Paris, Seuil.

Bayard, Pierre (2008), L'Affaire du chien des Baskerville, Paris, Éditions de Minuit, « Paradoxe ».

Blumenberg, Hans (2005), La Raison du mythe, trad. de Stéphane Dirschauer, Paris, Gallimard, «Bibliothèque de philosophie ».

Brunel, Pierre (1988), Dictionnaire des mythes littéraires, Monaco, Éditions du Rocher.

Boucher, François-Emmanuël, Sylvain David \& Maxime Prévost (dir.) (2014), Mythologies du superhéros. Histoire, physiologie, géographie, intermédialités, Liège, Presses Universitaires de Liège, «ACME ». 
Burke, Edmund (1968 [1757]), A Philosophical Enquiry into the Origin of our Ideas of the Sublime and the Beautiful, éd. de James T. Boulton, Notre Dame/London, University of Notre Dame Press.

Cassirer, Ernst (1993), Le Mythe de l'État, trad. Bertrand Vergely, Paris, Gallimard.

Castoriadis, Cornelius (1975), L'Institution imaginaire de la société, Paris, Éditions du Seuil, « Points essais ».

Detienne, Marcel (1981), L'Invention de la mythologie, Paris, Gallimard, « Bibliothèque des sciences humaines».

Conan Doyle, Arthur (2001 [1912]), The Lost World, éd. de Philip Gooden, Londres, Penguin Books. Duchet, Claude \& Patrick Maurus (2011), Un cheminement vagabond, Paris, Honoré Champion.

Dupuy, Lionel (2013), Jules Verne. La géographie et l'imaginaire, Dole, La Clef d'Argent.

Eliade, Mircea (1973), Fragments d'un journal, trad. du roumain par Luc Badesco, Paris, Gallimard, « Du monde entier».

Guillaud, Lauric (2014), Des mines du roi Salomon à la quête du Graal. H. R. Haggard (1856-1925), Paris, Michel Houdiard.

Haggard, Henry Rider (2001 [1887]), She, éd. de Patrick Brantlinger, Londres, Penguin Classics.

Jauss, Hans Robert (1988), Pour une herméneutique littéraire, trad. Maurice Jacob, Paris, Gallimard, «Bibliothèque des idées».

Pinson, Guillaume \& Maxime Prévost (dir.) (2019), Jules Verne et la culture médiatique. De la presse du XIX siècle au steampunk, Québec, Presses de l'Université Laval, « Littérature et imaginaire contemporain ».

Prévost, Maxime (2018), Alexandre Dumas mythographe et mythologue. L'Aventure extérieure, Paris, Honoré Champion, « Romantisme et modernités ».

Seillan, Jean-Marie (2008), « Petite Histoire d'une révolution épistémologique : la captation de l'héritage d'Alexandre Dumas par Jules Verne », dans Corinne Saminadayar-Perrin (dir.), Qu'est-ce qu'un événement littéraire au XIX siècle ?, Publications de l'Université de Saint-Étienne, p. 199-218.

Taves, Brian (2015), Hollywood Presents Jules Verne. The Father of Science Fiction on Screen, Lexington, University Press of Kentucky.

Verne, Jules (2016 [1864]), Voyage au centre de la terre, éd. de Jean-Luc Steinmetz, Paris, Gallimard, «Bibliothèque de la Pléiade»

Vial, André (1974), « Ce qui restera de Dumas père... », Revue d'histoire littéraire de la France, cxxIV p. 1015-1031.

Wallace, Edgar \& Merian C. Cooper, novélisation par Delos W. Lovelace (2005 [1932]), King Kong, New York, Modern Library.

\section{NOTES}

1. «Ce que l'individu peut produire, c'est des phantasmes privés, non pas des institutions. La jonction s'opère parfois, de même façon que l'on peut situer et dater, chez les fondateurs de religions et autres "individus exceptionnels", dont le phantasme privé vient combler là où il faut et à point nommé le trou de l'inconscient des autres, et possède suffisamment de "cohérence" 
fonctionnelle et rationnelle pour s'avérer viable une fois symbolisé et sanctionné - c'est-à-dire institutionnalisé » (Castoriadis $1975: 218-219)$.

2. Je condense dans cette section une matière développée plus avant dans Prévost 2018.

3. Cf. à la même page: "Les mythes, c'est l'histoire sainte des autres ». Angot s'inspire ici partiellement de Marcel Detienne (1981).

4. C'est ce que Pierre Bayard appelle le «complexe de Holmes": "Je propose d'appeler "complexe de Holmes" la relation passionnelle conduisant certains créateurs ou certains lecteurs à donner vie à des personnages de fiction et à nouer avec eux des liens d'amour ou de destruction » $(2008: 124)$.

5. Voir Vial $1974: 1015$.

6. Voir Boucher, David et Prévost (2014).

7. Voir Taves $2015: 9$.

8. Au sens de Castoriadis ; «l'essentiel de la création n'est pas "découverte”, mais constitution du nouveau: l'art ne découvre pas, il constitue; et le rapport de ce qu'il constitue avec le "réel", rapport assurément très complexe, n'est en tout cas pas un rapport de vérification » (1975:200).

9. Voir Jauss 1988, notamment p. 219 : «L'histoire littéraire d'un mythe n'est plus une sorte de monologue, où s'exprime progressivement un sens préexistant dans sa pureté et sa plénitude originelles, mais une sorte de dialogue, qui devient une appropriation croissante d'œuvre en œuvre à travers l'histoire d'une réponse à une grande question qui touche tout à la fois l'homme et le monde ; cela étant, avec chaque nouvelle formulation de la question, la réponse peut avoir encore un autre sens. »

10. Voir Duchet et Maurus : «Le cotexte est ce qui dans le texte ouvre à un en-dehors du texte, sur un ailleurs du texte, sur un domaine avec lequel le texte travaille. Avec lequel tout texte travaille. [...] Le cotexte n'est pas la totalité de l'univers, il est la portion de l'univers avec laquelle le texte travaille » (2011:44-45).

11. Castoriadis utilise souvent l'expression "collectif anonyme" sans la définir clairement. Rappelons que pour ce philosophe, l'imaginaire est instituant par l'activité d'un collectif anonyme. L'imaginaire instituant ne nous apparaît ainsi que dans ses créations: les formes successives instituées par un collectif anonyme. Voir «Les Significations imaginaires sociales » (1975 : 493-538, p. 533, notamment).

12. Je synthétise ici des considérations développées plus longuement dans Pinson et Prévost (2019: 16-19 et $157-161)$.

13. Verne 2016 [1864] : 180.

14. Verne 2016 [1864] : 182.

15. Sur cet auteur, voir Guillaud 2014.

16. Challenger : "It was surely well for man that he came late in the order of creation. There were powers abroad in earlier days which no courage and no mechanism of his could have met. What could his sling, his throwing-stick, or his arrow avail him against such forces as have been loose tonight? Even with a modern rifle it would be all odds on the monster » (Conan Doyle 2001 [1912] : 122).

17. Conan Doyle 2001 [1912] : 194. Cf. les propos du rabat-joie Tarp Henry, qui tente de dissuader Malone d'accompagner le professeur Challenger : « My dear chap, things don't happen like that in real life. People don't stumble upon enormous discoveries and then lose their evidence. Leave that to the novelists " (2001 [1912] : 45).

18. Voir Edmund Burke 1968 [1757] : 39 : "Whatever is fitted in any sort to excite the ideas of pain, and danger, that is to say, whatever is in any sort terrible, or is conversant about terrible objects, or operates in a manner analogous to terror, is a source of the sublime; that is, it is productive of the strongest emotion which the mind is capable of feeling. »

19. Voir Wallace, Cooper et Lovelace 2005 [1932] : XxII.

20. Guillermin, John (réalisation) (1976), King Kong, Paramount Pictures. 
21. Jackson, Peter (réalisation) (2005), King Kong, Universal Pictures.

22. Vogt-Robert, Jordan (réalisation) (2017), Kong : Skull Island, Warner Bros., 2017.

23. De même, une historicisation sur le plus long terme de la mythologie du monde perdu ne pourrait faire l'économie d'une étude de la franchise cinématographique Jurassic Parc / The Lost World.

\section{RÉSUMÉS}

Cet article s'intéresse à la caution donnée par le collectif anonyme à une topique qui, en quelques décennies, s'est transformée en mythologie : celle du «monde perdu », c'est-à-dire cette topique à l'origine romanesque (Voyage au centre de la terre, 1864) du monde primordial, enfoui, oublié, débouchant sur un temps différent radicalement du temps historique qui est le nôtre, topique qui, dans les décennies de la première réception de l'œuvre de Verne, s'institue avec tout le caractère de l'évidence propre aux mythologies modernes, transitant notamment par The Lost World d'Arthur Conan Doyle (1912) et King Kong de Merian C. Cooper (1933). Il est suggéré que cette mythologie des mondes perdus, loin d'être le seul "phantasme privé d'un individu exceptionnel » (Cornelius Castoriadis), est parvenue pénétrer l'imaginaire collectif parce qu'elle objectivait un questionnement collectif sur le confort moderne et l'existence sécuritaire : l'être humain est-il encore à la hauteur du sublime de sa planète, dans un monde où le mystère et les espaces vierges sur les cartes géographiques se raréfient?

This paper studies a literary commonplace which, over the course of a few decades, became a modern mythology : that of the "lost world ", that is the idea, originating from romance (Jules Verne's Journey to the Center of the Earth, 1864) of a primordial, buried, forgotten world stemming from an alternative timeline to our historic time. This topic became mythology, transiting though Arthur Conan Doyle's The Lost World (1912) and Merian C. Cooper's King Kong (1933). It is argued that this lost world mythos, far from being the «private fantasy of an exceptional individual» (to quote Cornelius Castoriadis), successfully penetrated the collective imaginary because it laid bare a largely unconscious collective unease about modern comfort and security : are human beings still worthy of their awe-inspiring planet?

INDEX

Mots-clés : mondes perdus, mythologies modernes, imaginaire social, Jules Verne, Arthur Conan Doyle, King Kong

Index géographique : Europe, Amérique, France, Royaume-Uni, États-Unis

Index chronologique : XIXe siècle, XXe siècle, 1864-1933

\section{AUTEUR}

\section{MAXIME PRÉVOST}

Maxime Prévost est professeur titulaire au Département français de l'Université d'Ottawa. Auteur de Rictus romantiques. Politiques du rire chez Victor Hugo (Presses de l'Université de Montréal, 2002) 
et de L'Aventure extérieure. Alexandre Dumas mythographe et mythologue (Paris, Honoré Champion, 2018), il s'intéresse à la littérature romantique et aux mythologies modernes. Il codirige avec François-Emmanuël Boucher la collection « Littérature et imaginaire contemporain » aux Presses de l'Université Laval. 